\title{
Tailored lighting intervention improves measures of sleep, depression, and agitation in persons with Alzheimer's disease and related dementia living in long-term care facilities
}

This article was published in the following Dove Press journal:

Clinical Interventions in Aging

12 September 2014

Number of times this article has been viewed

\author{
Mariana G Figueiro' \\ Barbara A Plitnick' \\ Anna Lok' \\ Geoffrey E Jones' \\ Patricia Higgins ${ }^{2,3}$ \\ Thomas R Hornick ${ }^{3,4}$ \\ Mark S Rea' \\ 'Lighting Research Center, Rensselaer \\ Polytechnic Institute, Troy, NY, \\ USA; ${ }^{2}$ School of Nursing, ${ }^{3}$ School \\ of Medicine, Case Western Reserve \\ University, ${ }^{4}$ Geriatric Research \\ Education and Clinical Center, Louis \\ Stokes Cleveland Veterans Affairs \\ Medical Center, Cleveland, OH, USA
}

Correspondence: Mariana G Figueiro Lighting Research Center, Rensselaer Polytechnic Institute, 2 I Union Street, 3rd floor, Troy, NY 12180 USA

$\mathrm{Tel}+\mathrm{I} 5186877100$

$\mathrm{Fax}+15186877120$

Email figuem@rpi.edu
Background: Light therapy has shown great promise as a nonpharmacological method to improve symptoms associated with Alzheimer's disease and related dementias (ADRD), with preliminary studies demonstrating that appropriately timed light exposure can improve nighttime sleep efficiency, reduce nocturnal wandering, and alleviate evening agitation. Since the human circadian system is maximally sensitive to short-wavelength (blue) light, lower, more targeted lighting interventions for therapeutic purposes, can be used.

Methods: The present study investigated the effectiveness of a tailored lighting intervention for individuals with ADRD living in nursing homes. Low-level "bluish-white" lighting designed to deliver high circadian stimulation during the daytime was installed in 14 nursing home resident rooms for a period of 4 weeks. Light-dark and rest-activity patterns were collected using a Daysimeter. Sleep time and sleep efficiency measures were obtained using the rest-activity data. Measures of sleep quality, depression, and agitation were collected using standardized questionnaires, at baseline, at the end of the 4-week lighting intervention, and 4 weeks after the lighting intervention was removed.

Results: The lighting intervention significantly $(P<0.05)$ decreased global sleep scores from the Pittsburgh Sleep Quality Index, and increased total sleep time and sleep efficiency. The lighting intervention also increased phasor magnitude, a measure of the 24-hour resonance between light-dark and rest-activity patterns, suggesting an increase in circadian entrainment. The lighting intervention significantly $(P<0.05)$ reduced depression scores from the Cornell Scale for Depression in Dementia and agitation scores from the Cohen-Mansfield Agitation Inventory.

Conclusion: A lighting intervention, tailored to increase daytime circadian stimulation, can be used to increase sleep quality and improve behavior in patients with ADRD. The present field study, while promising for application, should be replicated using a larger sample size and perhaps using longer treatment duration.

Keywords: sleep disorders, light therapy, circadian rhythms, ADRD

\section{Introduction}

Alzheimer's disease and related dementia (ADRD) is the most common mental disorder diagnosed in elderly Americans, with an estimated 5.1 million people affected in $2010 .{ }^{1}$ Behavioral symptoms, such as disturbed sleep-wake patterns, nocturnal wandering, agitation, and physical or verbal abuse, are among the most prevalent reasons that individuals with ADRD transition to more controlled environments. 
Light therapy has shown great promise as a nonpharmacological method to improve behavioral symptoms associated with ADRD. Several studies have found that exposure to bright white light (at least 2,500 lux and as high as 8,000 lux at the cornea) for at least 1 hour in the morning for a period of at least 2 weeks consolidated rest-activity patterns in ADRD patients, resulting in greater nighttime sleep duration and sleep efficiency, and more wakefulness during daytime hours. ${ }^{2-4}$ Unattended exposure to bright white light $(>1,000$ lux at the cornea) during the entire day was shown to improve rest-activity patterns of ADRD patients. ${ }^{5}$ Data from Riemersma-van der Lek et $\mathrm{al}^{6}$ showed, in the largest randomized placebocontrolled, double-blind study ever conducted with this population, that high levels of unattended exposures to white light $(>2,500$ lux at the cornea) could not only improve sleep but also slow down cognitive decline in ADRD patients.

One study failed to show an effect of light therapy on objective measures of nighttime sleep, unless patients had aberrant timing of their rest-activity rhythms. ${ }^{7}$ Another study showed that bright light therapy modestly reduced duration of nighttime awakening, without significantly affecting the percentage of nighttime sleep or number of nighttime awakenings. ${ }^{8}$

Light therapy has also been shown to reduce agitation behavior in this population..$^{2,39-11}$ Evening exposure to bright white light $(>1,000$ lux at the cornea) for 2 hours was shown to decrease nocturnal activity and the severity of evening agitation ("sundowning") of ADRD patients. ${ }^{12}$ However, other studies failed to show the positive effects of light therapy on agitation and behavioral disturbances in ADRD patients; in some cases, light changed the circadian phase or improved nighttime sleep, ${ }^{3,13}$ or even showed an increase in agitated behavior with morning light. ${ }^{14}$

Colenda et a ${ }^{15}$ investigated the impact of a light visor on the sleep patterns of community-dwelling subjects and failed to find a consistent biological effect of light on the subjects. The authors suggested that the light delivery method may not have been very successful in delivering the appropriate dose to the subjects' eyes. Fontana Gasio et $\mathrm{al}^{16}$ investigated whether a low-intensity dawn-dusk simulator - a halogen lamp with light levels varying from 0.01 to 200 lux at the level of the cornea - could improve circadian rhythm disturbances in ADRD patients. The authors did not show any significant changes in cognitive status or rest-activity patterns. Compared with a dim red light control condition (authors reported using a $15 \mathrm{~W}$ red light bulb yielding $<5$ lux), they were only able to demonstrate that the dawn simulator resulted in significant earlier sleep episodes and in a nonsignificant tendency to shorter sleep latency, longer sleep duration, and less nocturnal activity.
Despite some inconsistencies, the protocols used by van Someren et al, ${ }^{5}$ Riemersma-van der Lek et $\mathrm{al}^{6}$ and Sloane et $\mathrm{a}{ }^{17}$ which employed prolonged, high light levels of white light ( $>1,000$ lux at the cornea), have produced positive outcomes. The disadvantages associated with this basic approach are the increased operating costs in the facilities and the eye discomfort resulting from high light levels. Recent research has shown that the human circadian system is maximally sensitive to short-wavelength (blue) light, with peak wavelength close to 460 nanometers (nm) ${ }^{18-20}$ This finding opens the door for the potential application of lower, more targeted light levels in therapeutic settings. Using this knowledge, two pilot studies showed that evening exposure to 2 hours of short-wavelength light (30 lux at the cornea) from light-emitting diodes (LEDs) peaking at $470 \mathrm{~nm}$ consolidated rest-activity rhythms and increased the sleep efficiency of persons with ADRD..$^{21,22}$ However, delivering light to ADRD patients via light boxes or light goggles is a challenge and would likely have low compliance rates.

A logical compromise solution would be to illuminate the occupied room with a white light source with a high proportion of short-wavelength radiation delivering lower corneal photopic light levels than those previously employed. ${ }^{5,6,17,23}$ Although studies have shown that short-wavelength light or "blue-enriched" light sources can be effective at correcting circadian sleep disorders, ${ }^{24,25}$ other studies failed to show differences between lamps of different correlated color temperatures (CCTs). Smith and Eastman ${ }^{26}$ compared the effectiveness of a 17,000 K lamp and a 4,100 K lamp in phase shifting the human circadian clock, under controlled laboratory conditions. The researchers did not find any significant differences between the light sources, most likely because they were using light levels above the saturation response of the circadian system. In fact, calculations performed using the model of human circadian phototransduction by Rea et $\mathrm{a}^{19}$ showed that both light sources should have the same impact on acute melatonin suppression. Therefore, from the studies to date, it has not been empirically demonstrated that white light sources with more short-wavelength content delivered at lower light levels than the ones used in previous studies can be as or more efficacious at treating circadian sleep disorders in ADRD patients.

The present study was designed to investigate the efficacy and feasibility of a tailored lighting intervention designed to deliver high circadian stimulation at moderate light levels from a high-CCT white light source. ${ }^{19}$ The lighting intervention illuminated the occupied room during daytime hours and was energized for 8-10 hours 
per day. The combined effect of a high-CCT lamp delivering moderately high light levels was calculated to be 40 times more effective at stimulating the circadian system than the lighting conditions commonly found in facilities for the elderly, where low-CCT sources deliver low light levels. Importantly, the plug-in luminaires used in the present study were inexpensive and easy to install. It was hypothesized that the lighting intervention would improve objective and subjective measures of sleep as well as reduce depression and agitation scores in those with ADRD.

\section{Materials and methods}

\section{Sample and setting}

Fourteen subjects (nine females; mean age was $86.9 \pm 4.4$ years) were recruited from long-term care facilities (skilled nursing homes) in the Albany, NY area. The mean Brief Interview for Mental Status score of the participants was $7.7 \pm 2.3$ - a score of $0-7$ suggests that residents are severely impaired, while a score of $8-12$ suggests that residents are moderately impaired. ${ }^{27}$ The attending physician and the primary caregivers identified potential participants who were observed to have sleep and agitation problems. The inclusion criteria for the study were: the ADRD elder's physician had to confirm a diagnosis of mild-moderate dementia, based on the Diagnostic and Statistical Manual of Mental Disorders, Fourth Edition (DSM-IV) criteria $^{28}$ and also agree that the ADRD elder was suitable for participation in the study. Subjects taking antidepressants were included; however, types of medicine and dosage intakes were monitored. There was no exclusion based on age, sex, race, or ethnicity. The exclusion criteria included major organ failure, major illness, history of head injury, or uncontrolled generalized disorders, such as hypertension or diabetes. Exclusion criteria also included the use of psychotropic (sleep aid) medicine, obstructing cataracts, macular degeneration, and blindness - the results of participants' ophthalmologic tests were reviewed prior to acceptance into the study. All study materials and procedures were reviewed and approved by the Institutional Review Board at Rensselaer Polytechnic Institute, Troy, NY. Informed consent was obtained from participant family members after full explanation of the procedures, in accordance with the Declaration of Helsinki. ${ }^{29}$

\section{Lighting intervention}

Custom luminaires, designed to illuminate the occupied room, were built for the study, using parts currently available on the market. Two GE 45851 F55BX/AR/FS fluorescent lamps (GE Lighting, Cleveland, OH, USA) were inserted in a luminaire head (ETC 454 Line Voltage T5 Fluorescent Wall Washer;

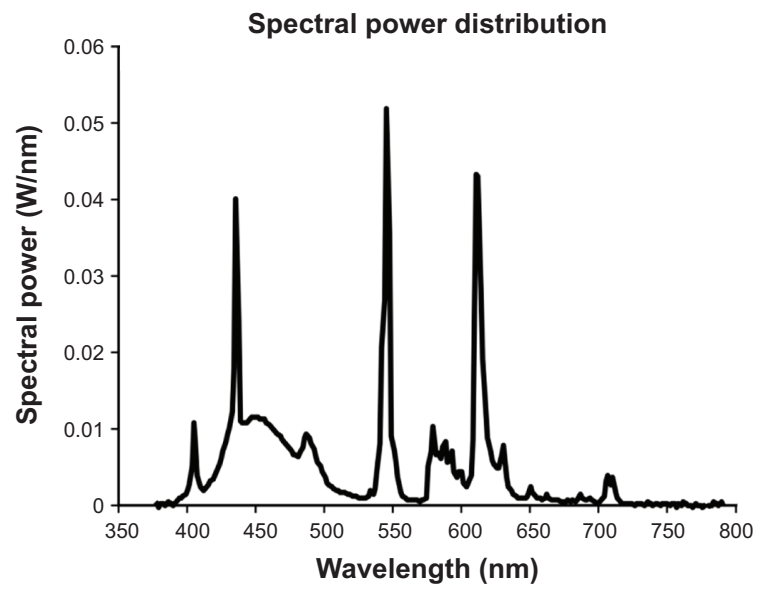

Figure I Spectral power distribution of the light source used in the study. Notes: The measured CCT of the light source was $9,325 \mathrm{~K}$. Abbreviation: CCT, correlated color temperature.

ELCO Lighting, Vernon, CA, USA). Figure 1 shows the spectral power distribution of the light source used in the study. The measured CCT of the light source was 9,325 K. To save energy, all luminaires were plugged into a GE 15079v2 SunSmart ${ }^{\mathrm{TM}}$ Digital Timer (GE Lighting). This timer was programmed to automatically turn on all luminaires close to the time each resident woke up (generally between 6-8 am) and off at $6 \mathrm{pm}$. During the day, when the luminaires were turned on, an additional layer of control was added by installing a passive infrared (PIR) motion sensor (OSFHU-ITW High Bay Occupancy Sensor; Leviton Manufacturing Co., Inc., Melville, NY, USA) directly onto each luminaire, automatically turning the lamps off after 20 minutes without detection of occupant movement. The luminaires were energized from a standard $120 \mathrm{VAC}$ wall power supply through a carefully concealed extension cord. The luminaire was affixed to a hinged gimbal on an $86 \mathrm{~cm}$ tall microphone stand; a quick release on the stand could extend the pole to $157 \mathrm{~cm}$. During installation, two $2.25 \mathrm{~kg}$ sandbag weights were also wrapped around the base, to prevent tipping. In order to minimize glare, the luminaire was tilted to direct light upward to the ceiling. Figure 2 shows an example of an installation in a subject's room.

The model of human circadian phototransduction proposed by Rea et $a{ }^{19}$ was used to estimate the circadian stimulus (CS) of the lighting intervention. While melatonin levels were not collected, calculations showed that 300-400 lux (at the cornea) of the high-CCT light would result in at least $50 \%$ melatonin suppression for a 1-hour exposure for a young subject, ${ }^{30}$ indicating that the light source used in the intervention delivered strong circadian stimulation. Although it is not known whether the CS for circadian entrainment and phase shifting is the same as the CS for acute melatonin suppression, 


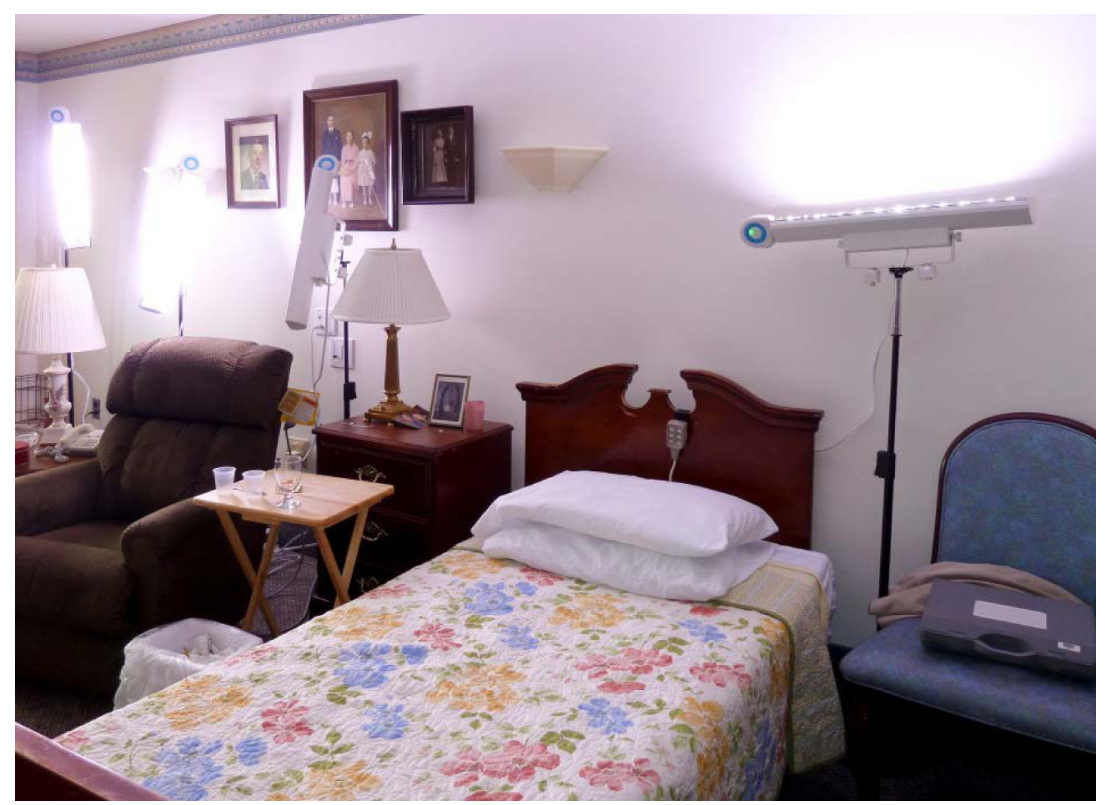

Figure 2 An example of luminaires installed in a subject's room for the lighting intervention.

Notes: The tailored lighting intervention used two GE 45,85I F55BX/AR/FS fluorescent lamps (GE Lighting, Cleveland, OH, USA) inserted in a luminaire head (ETC 454 Line Voltage T5 Fluorescent Wall Washer; ELCO Lighting, Vernon, CA, USA). Luminaires were automatically turned on between 6-8 am and off at 6 pm by a timer, and turned on during the day, via an infrared occupancy sensor, only when the room was occupied.

the literature suggests that these responses to light are similar. ${ }^{31}$ Due to changes in the aging eye, older people are slightly less sensitive to short wavelengths than are young observers for any source of light, but the differential effect can be estimated. The optical density of a normal 60-year-old person's crystalline lens is about 0.2-fold greater at short wavelengths than that of a 20-year-old; thus, for a normal 60-year-old, the relative crystalline lens transmission at short wavelengths would be $63 \%$ that of a normal 20 -year-old. This age-dependent differential density of the lens at short wavelengths is comparable with having the 60 -year-old observer view a blue light source $25 \%$ closer than for the 20-year-old observer (eg, 2.25 vs $3 \mathrm{~m}$ ). Taking lens transmission into consideration, the intervention was predicted to deliver a CS of 0.375 , which was based upon a measured melatonin suppression of $37.5 \%$ after 1-hour exposure during the night. Since the intervention was delivered for a period longer than 1 hour per day, the overall circadian light dose was inevitably increased.

\section{Outcome measures}

The outcome measures included objective measures of sleep, rest-activity patterns, and circadian disruption, using data taken from a Daysimeter (Lighting Research Center, Rensselaer Polytechnic Institute, Troy, NY, USA); and subjective measures of sleep quality, depression, agitation, and activities of daily living, using standardized questionnaires. Data were collected 1) prior to the lighting intervention installation (baseline), 2) at the end of the 4-week lighting intervention (intervention period), and 3) 4 weeks after the lighting intervention was removed (post-intervention period).

\section{Daysimeter}

The Daysimeter is a small device that continuously records light (using red, green, and blue [RGB] sensors) and activity levels. ${ }^{32}$ Upon downloading, the RGB values are converted into illuminance (lux), circadian light $\left(\mathrm{CL}_{\mathrm{A}}\right)$, and CS levels. ${ }^{33}$ Briefly, illuminance is irradiance weighted by the photopic luminous efficiency function, $\mathrm{V}(\lambda)$, an orthodox measure of the spectral sensitivity of the human fovea, peaking at $555 \mathrm{~nm} . \mathrm{CL}_{\mathrm{A}}$ is irradiance weighted by the spectral sensitivity of the retinal phototransduction mechanisms stimulating the response of the biological clock, based on nocturnal melatonin suppression. $\mathrm{CS}$ is a transformation of $\mathrm{CL}_{\mathrm{A}}$ into relative units, from 0 (the threshold for circadian system activation) to 0.7 (response saturation), and is directly proportional to nocturnal melatonin suppression after 1-hour exposure (0\%-70\%).

Rea et $\mathrm{al}^{34}$ have proposed a quantitative technique to measure circadian disruption, known as phasor analysis, which quantifies circadian disruption in terms of the phase and the amplitude relationship between the measured light-dark stimulus pattern and measured activity-rest response pattern. Phasor analysis makes it possible to interpret the light and activity data, sampled together over consecutive multiple days. To quantify circadian disruption using the Daysimeter data, we used the measured circadian light-dark pattern and restactivity pattern. The relationship between these two sets of 
time-series data was quantified through phasor analysis, which integrates a discrete Fourier transform power and phase analysis of the circular correlation function computed from the two data sets (light-dark and activity-rest data). A cosine curve was fitted to each of the activity-rest and light-dark patterns, using least squares. The phasor angle was determined by the phase difference between each of the fitted cosine curves, and the phasor magnitude was the normalized cross-correlation of the fitted cosine curves and the light and activity data. The resulting vector or phasor quantified, in terms of the 24-hour frequency, how closely tied the light and activity patterns were to a 24-hour pattern (phasor magnitude) as well as their relative temporal relationship (phasor angle).

Phasor analysis was used to characterize the relationship between the 24-hour light-dark pattern and the 24-hour restactivity pattern. ${ }^{34}$ Since CS is a measure of the effectiveness of optical radiation on the retina in stimulating the human circadian system, the daily patterns of CS were used in the phasor analyses - the larger the phasor magnitude, the greater the correlation between these two rhythms.

In addition, the activity data from the Daysimeter were used to calculate interdaily stability (IS) and intradaily variability (IV). The IS quantified the extent to which all recorded 24-hour activity profiles resemble each other, which represented the day-by-day regularity of the sleep-wake pattern. The IV quantified the fragmentation of the rhythm, that is, the frequency and extent of transitions between periods of rest and activity. ${ }^{5}$

\section{Sleep analyses}

The activity data from the Daysimeter were also used to obtain estimates of sleep parameters, including total sleep time, sleep efficiency (percentage of actual sleep between lights out and final awakening), and sleep-onset latency (the time between lights out and sleep onset).

\section{Standardized questionnaires}

Four questionnaires were administered to assess subjective sleep quality, depression, activity of daily living, and agitation:

1. Pittsburgh Sleep Quality Index (PSQI): ${ }^{35}$ the PSQI is an instrument that can be used to measure sleep quality in clinical populations and is composed of 19 items that generate seven component scores (subjective sleep quality, sleep latency, sleep duration, habitual sleep efficiency, sleep disturbances, use of sleep medication, and daytime dysfunction). The sum of the seven component scores yields one global score. A person with a global score above 6 is considered to have sleep disturbances.
2. Minimum Data Set Activities of Daily Living Scale (MDS-ADL): ${ }^{36}$ the MDS-ADL measures activities related to personal care and includes bathing, dressing, getting in or out of bed or a chair, using the toilet, and eating. A higher score is associated with greater dependence in performance of personal care.

3. Cornell Scale for Depression in Dementia (CSDD): ${ }^{37}$ the CSDD is a 19-item tool designed to rate symptoms of depression in persons with dementia. This tool evaluates the presence and extent of mood-related signs (anxiety, sadness, irritability), behavioral disturbances (agitation, loss of interest), physical signs (loss of appetite, weight loss), cyclic functions (mood variation, sleep quality), and ideational disturbances (suicidal thoughts, poor self-esteem). A higher self-report score is associated with greater depression, a score of 12 or higher is considered to indicate depression.

4. Cohen-Mansfield Agitation Inventory (CMAI): ${ }^{38}$ the CMAI is a caregivers' rating questionnaire consisting of 29 agitated behaviors, each rated on a seven-point scale designed to assess the frequency of manifestations of agitated behaviors in elderly persons. A higher CMAI is associated with greater agitation.

\section{Experimental protocol}

The facility physician and administrator identified potential subjects for the study, based on the exclusion criteria listed above. Only the residents who tended to stay in their rooms often (rather than participate in other activities outside their rooms) were included in the study. The facility administrator obtained consent from family members. Once enrolled in the study, the research nurse worked with the nighttime caregiver to obtain the baseline assessment, which included the questionnaires and the Daysimeter data collection. Site spot light measurements were also obtained at baseline. Baseline light measurements were taken with an illuminance meter (Gigahertz-Optik X9 ${ }_{1}$ Photometer; GigahertzOptik GmbH, Türkenfeld, Germany), placed vertically at eye level where residents were normally seated. The window shades in the rooms were pulled down to remove daylight. After baseline data collection, the research nurse and research assistant installed the lights in the residents' rooms. The number of luminaires installed and location of the luminaires were determined based on the room size and where residents spent most of their time during the day. The luminaires were programmed by a timer to be turned on at the time each participant awoke (wake times were provided by daytime caregivers) and turned off at $6 \mathrm{pm}$ daily. At the end of the fourth week, the luminaires were removed from the residents' bedrooms. The post-intervention data 
collection was performed 4 weeks after the luminaires were removed from the residents' rooms.

The Daysimeters were deployed for a period of 1 week, at baseline and at the end of the third week after the lighting intervention was initiated. Post-intervention Daysimeter data were collected but due to low compliance rates, they were not evaluated and are not discussed here. Data were collected between December and March and between April and September, to accommodate for daylight savings time changes.

The caregivers were instructed to ensure the Daysimeter device was worn continuously (24 hours/day, except when showering) by the subjects for the entire data collection period. The device was placed on the participants' wrists and caregivers were instructed to ensure blankets and long-sleeve sweaters and shirts did not cover it. The primary caregivers (those working nightshifts) were asked to complete the standardized questionnaires at baseline, at the end of the fourth week of the lighting intervention, and at the end of the fourth week after the luminaires were turned off and removed from the rooms (post-intervention). While it was not possible to completely blind the caregivers from the experimental conditions, none of the caregivers were informed about the goals of the study until the end of the experiment, when a debrief was offered. The only staff members aware of the goals of the study were the physicians, the daytime charge nurse, and the administrator, who obtained consents from the families.

Researchers interviewed daytime caregivers, but no formal data were collected from them, as the goal of the present study was to investigate the effect of the lighting intervention on nighttime sleep and agitation. Although caregivers completed the questionnaires for most of the subjects, for some subjects, the caregiver did not complete one or all the questionnaires; therefore, some data were missing, as noted in the results section below.

\section{Results}

\section{Site illuminance measurements}

The measured mean \pm standard deviation (SD) baseline light level at the cornea prior to the intervention was $66 \pm 130$ lux ( median $=32$ lux). Another light measurement was performed after the four floor lamps were installed in each room for the lighting intervention. The mean \pm SD light levels at the cornea obtained after the luminaires were installed in the rooms was $324 \pm 190$ lux (median $=324$ lux).

\section{Daysimeter data}

A total of ten subjects wore the Daysimeter. Data for 7 consecutive days were usable from six subjects, data for 4 consecutive days were usable from two subjects, and data for 3 consecutive days were usable from two subjects. The mean \pm standard error of the mean (SEM) $\mathrm{CL}_{\mathrm{A}}$ during the waking period (removing the data from staff-reported bedtimes and wake times) was $103 \pm 31$ (median =62) at baseline and $373 \pm 121$ (median $=266$ ) during intervention. A two-tailed paired $t$-test revealed statistical significance between circadian light exposures at baseline and during intervention $(P=0.046)$.

The mean \pm SEM CS (calculated using data from when subjects were awake) was $0.06 \pm 0.01$ (median $=0.04)$ at baseline and $0.1 \pm 0.01$ during intervention (median $=0.1$ ). Although the CS values obtained from wrist measurements were much lower than those calculated from spot measurements using an illuminance meter were made, a two-tailed paired $t$-test revealed a significant difference between $C S$ at baseline and during intervention $(P=0.0003)$. Nevertheless, as discussed above, the onsite spot measurements using a commercial illuminance meter showed that the intervention irradiance levels were fivefold higher than those at baseline.

The mean $\pm \mathrm{SEM}$ phasor magnitude was $0.27 \pm 0.03$ at baseline and $0.35 \pm 0.02$ during intervention. A two-tailed paired $t$-test revealed a significant increase in phasor magnitude during intervention ( $P=0.001)$ compared with baseline. An increase in phasor magnitude suggests an increase in circadian entrainment. ${ }^{34}$ For comparison, in one study, healthy older adults had a mean phasor magnitude of $0.4{ }^{39}$

The average light (CS) and activity (AI) profiles for all subjects at baseline and during intervention are shown in Figures 3A and B, respectively. It can be clearly seen from these graphs that daytime CS during intervention was much higher than that observed at baseline. There was also an increase in daytime activity, especially after lunch.

There were no significant differences between IS and IV values at baseline and during intervention $(P>0.05)$. Mean \pm SEM IS scores were $0.73 \pm 0.06$ at baseline and $0.74 \pm 0.05$ during intervention. Mean \pm SEM IV scores were $0.62 \pm 0.06$ at baseline and $0.63 \pm 0.07$ during intervention.

\section{Sleep analyses}

Mean \pm SEM sleep efficiency was $80 \% \pm 5 \%$ at baseline and $84 \% \pm 4 \%$ during intervention, as shown in Figure 4. A two-tailed paired $t$-test revealed a significant increase in sleep efficiency $(P=0.03)$. As shown in Figure 5, mean \pm SEM total sleep time (in minutes) was $431 \pm 37$ at baseline and $460 \pm 25$ during intervention. A two-tailed paired $t$-test revealed a significant increase in sleep time during intervention $(P=0.03)$. Sleep latency was not significantly different at baseline and during intervention. Mean \pm SEM sleep latency (in minutes) was $36 \pm 14$ at baseline and $32 \pm 8$ during intervention. 
A

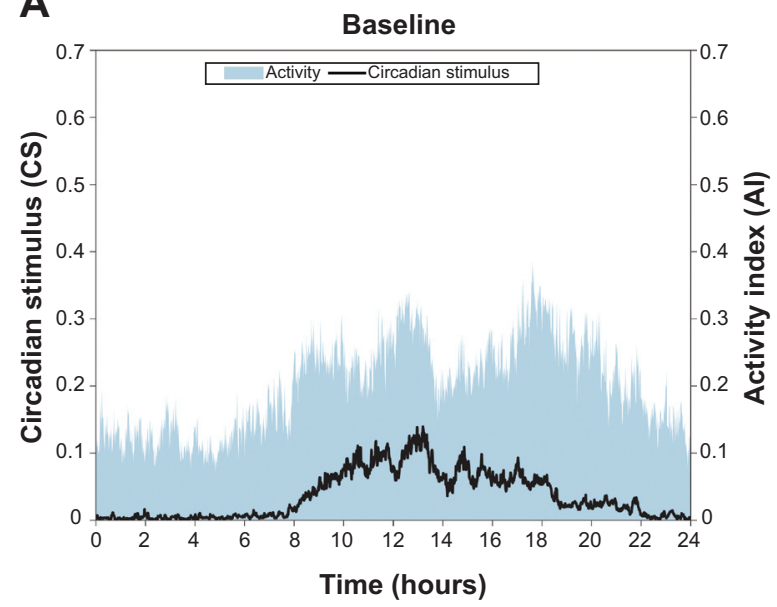

B

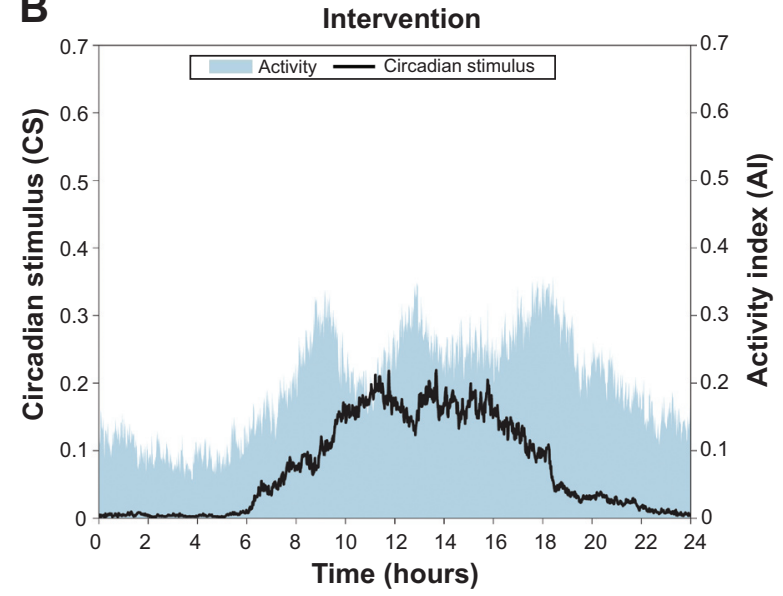

Figure 3 Average circadian stimulus (CS) and activity index (Al) for all subjects, at baseline $(\mathbf{A})$ and during intervention (B).

Notes: Both CS and activity increased during daytime hours during intervention.

\section{Standardized questionnaire data PSQI}

Ten subjects had fully completed questionnaires for the baseline and intervention periods, and eight subjects had fully completed questionnaires for the post-intervention period. As shown in Figure 6, the global PSQI scores were reduced during intervention. The mean \pm SEM of the global PSQI score at baseline was $8.7 \pm 1.5$. During the 4 -week lighting intervention, the mean \pm SEM PSQI score was $4.1 \pm 0.6$. A two-tailed paired $t$-test showed significantly lower PSQI scores during intervention than at baseline $(P=0.01)$. The post-intervention period score was $5.3 \pm 1.1$, suggesting that there was a slight carry over effect of the lighting intervention on PSQI scores (as previously described, a score of 6 or higher is considered an indication of sleep disturbances). It is interesting to note that although the PSQI scores were still lower 4 weeks after the lighting intervention was turned off, the SEM was higher, suggesting that the variance in sleep quality increased after the removal of the luminaires.

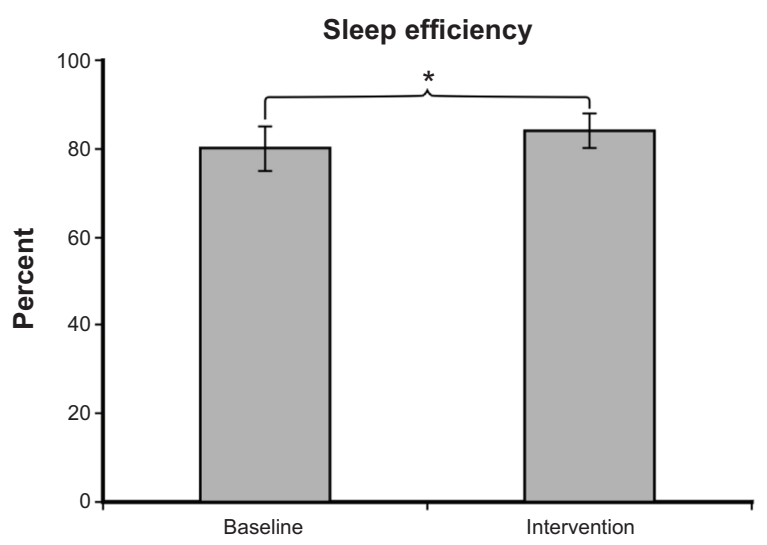

Figure 4 Mean \pm SEM sleep efficiency was $80 \% \pm 5 \%$ at baseline and $84 \% \pm 4 \%$ during intervention. Sleep efficiency during intervention was significantly greater than at baseline $(P=0.03)$.

Notes: Daysimeter data were not available for the post-intervention period due to poor compliance. $* P<0.05$.

Abbreviation: SEM, standard error of the mean

\section{MDS-ADL}

Fourteen subjects had fully completed questionnaires for the baseline and intervention periods, and 12 subjects had fully completed questionnaires for the post-intervention period. The mean \pm SEM MDS-ADL scores were $9.3 \pm 2.0$ at baseline, $9.1 \pm 2.0$ during intervention, and $7.4 \pm 1.7$ post-intervention. A two-tailed paired $t$-test did not show a significant difference between MDS-ADL scores at baseline and during intervention $(P=0.9)$.

\section{CSDD}

Thirteen subjects had fully completed questionnaires for the baseline, intervention, and post-intervention periods. As shown in Figure 7, depression scores were reduced during intervention compared with baseline measurements.



Figure 5 Mean \pm SEM total sleep time (in minutes) was $431 \pm 37$ at baseline and $460 \pm 25$ during intervention. Sleep time during intervention was significantly greater than at baseline $(P=0.03)$.

Note: Daysimeter data were not available for the post-intervention period due to poor compliance. $* P<0.05$.

Abbreviation: SEM, standard error of the mean. 


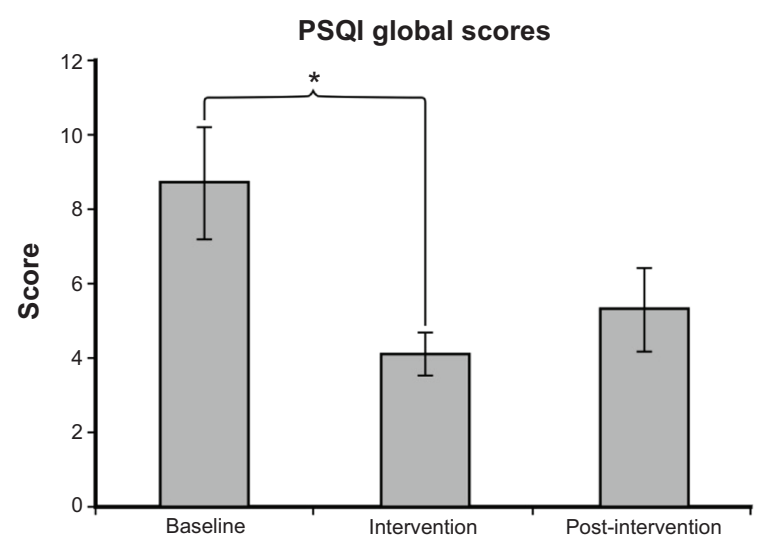

Figure 6 The mean \pm SEM of the global PSQI score was $8.7 \pm 1.5$ at baseline, $4.1 \pm 0.6$ during intervention, and $5.3 \pm \mathrm{l}$.I post-intervention. A significantly higher PSQI score was observed at baseline than during intervention $(P=0.0 \mathrm{I})$.

Notes: Scores $>6$ indicate sleep disturbances. $* P<0.05$.

Abbreviations: PSQI, Pittsburgh Sleep Quality Index; SEM, standard error of the mean.

The mean \pm SEM CSDD scores were 12.0 \pm 1.5 at baseline, $6.0 \pm 1.6$ during intervention, and $9.0 \pm 2.0$ post-intervention. A two-tailed $t$-test revealed a significant decrease in depression scores from baseline to intervention $(P=0.03)$.

\section{CMAI}

Fourteen subjects had fully completed questionnaires for the baseline and intervention periods, and 12 subjects had fully completed questionnaires for the post-intervention period. As shown in Figure 8, the lighting intervention resulted in a reduction in agitation scores. The mean \pm SEM of the CMAI score was $38.2 \pm 2.8$ at baseline, $31.2 \pm 0.7$ during intervention, and $32.3 \pm 1.1$ post-intervention. A two-tailed paired $t$-test revealed a significant difference between baseline and



Figure 7 The mean \pm SEM CSDD scores were $12.0 \pm 1.5$ at baseline, $6.0 \pm 1.6$ during intervention, and $9.0 \pm 2.0$ post-intervention. A significantly higher depression score was observed at baseline than during intervention $(P=0.03)$.

Notes: Higher scores are associated with greater self-report of depression, with depression being associated with scores of 12 or higher. $* P<0.05$.

Abbreviations: CSDD, Cornell Scale for Depression in Dementia; SEM, standard error of the mean.



Figure 8 The mean \pm SEM of the CMAI score was $38.2 \pm 2.8$ at baseline, $31.2 \pm 0.7$ during intervention, and $32.3 \pm 1.1$ post-intervention. A significantly higher CMAI score was observed at baseline than during intervention $(P=0.037)$ and post-intervention $(P=0.03)$.

Notes: $\mathrm{A}$ higher $\mathrm{CMAl}$ is associated with greater agitation. ${ }^{*} P<0.05$.

Abbreviations: CMAI, Cohen-Mansfield Agitation Inventory; SEM, standard error of the mean.

intervention scores $(P=0.037)$ and between baseline and post-intervention $(P=0.03)$. Agitation was one of the major complaints by the caregivers; informal interviews with the caregivers revealed that resident behavior was improved with the lighting intervention.

\section{Discussion}

The present study set out to investigate the effects of a tailored lighting intervention on sleep and on behavior in those with ADRD living in nursing homes. It was hypothesized that, because the circadian system is maximally sensitive to shortwavelength (blue) light, peaking close to $460 \mathrm{~nm}$, exposure to moderate levels of light with high short-wavelength content (bluish-white light) during the day would more positively impact objective and subjective sleep and behavior measures in persons with ADRD than would light sources with less shortwavelength content (yellowish-white light), commonly found in long-term care facilities at the same photopic illuminance. The present results showed that 300-400 lux of a bluish-white light significantly improved sleep efficiency and global PSQI scores, and decreased depression (CSDD) and agitation (CMAI) scores. Informal interviews with caregivers performed prior to starting the study revealed that agitation was one of the greatest issues they encountered when caring for the residents. The lighting intervention also increased phasor magnitude, suggesting an increase in circadian entrainment.

The questionnaire data need to be considered with caution for two reasons. The first is related to the use of unblinded professional caregivers to answer the questionnaires (by proxy), which may have introduced an element of bias. The caregivers may have known which arm the intervention was on and answered accordingly. However, this seems unlikely 
because the caregivers did not know the purpose of the study, were unfamiliar with the questionnaires, and their responses did not always favor the intervention group. For example, the MDS-ADL scale remained stable, which was expected given that physical functioning of older adults is influenced by multiple comorbid factors other than just sleep quality. In addition, the objective measures of sleep (efficiency and sleep times) revealed results consistent with the subjective ratings provided by caregivers. A second important methodological consideration was the use of proxy data. The choice of using the proxy data instead of self-report data stemmed from the fact that all participants were moderately to severely demented.

The CS values during the intervention obtained from Daysimeter measurements were low. A CS of 0.1 suggests that the lighting would suppress melatonin by about $10 \%$ after 1-hour exposure - this low value suggests that the Daysimeters were covered for most of the time that subjects were wearing them. Although Figueiro et $\mathrm{al}^{32}$ showed that placing the Daysimeter on the wrist may compromise the accuracy for estimating corneal light exposures, this was the only location that the device could be worn reliably by residents. Indeed, visual inspection of the data showed that the device was being covered often, despite that the experimenters reminded the caregivers about the importance of keeping the device uncovered. This was determined from looking at the activity and light data. While the recorded mean CS values were very low, the activity data were still high, indicating that the subjects were not asleep and yet, the recorded light levels were extremely low. Future studies should use two devices - a wrist actigraph for continuously monitoring rest-activity patterns and a pendant Daysimeter, which has been shown by Figueiro et $\mathrm{al}^{39}$ to correlate well with light at eye level.

Based on the spot measurements, light levels used in the present study were one-third of those used by van Someren et $\mathrm{al}^{5}$ and Sloane et al. ${ }^{17}$ Lower light levels can reduce facilities' operating costs and because the room can be diffusely illuminated, compliance should increase due to the reduction in glare, a common complaint among older adults. These quantities were selected based on calculations made using the mathematical model developed by Rea et al. ${ }^{19}$ According to the calculations, the spectrum of the light source used in the intervention provided a CS value of approximately fourfold that of an incandescent light source at the same photopic illuminance. In addition to the prescribed change in the intervention light source spectrum, the total irradiance from the intervention light source delivered approximately fivefold more irradiance than that used in the facilities. Thus, by combining spectrum and light level, the intervention lighting had the potential to produce a 20 -fold greater circadian stimulation than the baseline lighting. This increase in circadian stimulation coupled with an all-day light exposure probably was responsible for the positive effects on sleep and behavior. Moreover, the intervention lighting was turned off at $6 \mathrm{pm}$ daily, ensuring that residents were exposed to low circadian stimulation (based on spot measurements, the light level from the electric lighting, at the cornea, was approximately 66 lux) during evening hours. Given that the circadian system may be more concerned with contrast (ie, day:night ratio) than with absolute light exposures,${ }^{40}$ the lighting intervention used in this study was also beneficial in this regard.

The present field study, while promising for application, should be replicated using a larger sample size and perhaps using longer treatment duration (eg, 6 months) to determine whether implementation of this kind of lighting in elder care facilities can have a long-term effect on sleep and on behavior of those with ADRD, and therefore reduce caregiver burden. While it is hypothesized that the same lighting intervention can have similar results if applied to those living at home, further investigation is needed before these results can be extrapolated to less controlled environments. Those living at home may not always stay in one single room, and the fidelity of the intervention may be compromised by reduced light exposure duration or, potentially, light exposure at the wrong times. Moreover, those living at home are more likely to receive irregular light-dark patterns resulting from trips outdoors or from more irregular sleep schedules. In fact, a recent study by Sloane et $\mathrm{al}^{41}$ showed that a tailored lighting intervention similar to the one used in the present study improved sleep in caregivers of those with ADRD but did not change sleep or behavior in the ADRD patients. In their study, one day, but not multiple days, of continuous light exposure measurements using the Daysimeter were obtained, so it was not possible to determine whether there was a significant difference between circadian light exposures at baseline and during intervention. Finally, although our study was not designed to specifically investigate whether there are sex differences in light therapy responses, it would be interesting to further investigate whether men and women respond to light therapy differently. Hickman et $\mathrm{al}^{42}$ showed a significant sex and treatment interaction, where depressive symptoms were the lowest for women and highest for men when subjects received morning light exposures.

The number of persons with dementia is anticipated to more than double by $2050 .{ }^{43}$ Sleep disturbances are common 
in this population and lead to poor quality of life and increased burden on caregivers, especially those working nightshifts. Medications are widely used, but the side effects are significant. The possible societal benefits associated with improving the sleep quality of older adults may include a reduced number of falls, increasing cognition, and the delay in transition of older adults with ADRD to more controlled living environments. However, many current approaches to light therapy for reducing sleep disturbances in older adults do not consider the complete 24-hour light-dark pattern they experience, nor do they integrate light (and dark) treatment into a practical delivery system, thus compromising the therapeutic value. The present study demonstrates that a light delivery system that is tailored to increase circadian stimulation during the daytime hours and reduce it during the evening hours ${ }^{23}$ can improve sleep and reduce agitation in those with ADRD living in long-term care facilities, without increasing discomfort glare. Although yet to be investigated, light therapy coupled with other activities ${ }^{44}$ may have additional benefits, such as improved attention, orientation, and memory in residents, as well as reduction in caregiver stress and burden.

\section{Acknowledgments}

The study was funded by the National Institute on Aging (grant \# R01AG034157). Mary Beth Gotti (GE Lighting, Cleveland, $\mathrm{OH}$, USA) donated the lamps and ballasts used in the study.

The authors would like to thank Dr Guerman Ermolenko for helping with subject recruitment. Erin Plonka at Teresian House and Agnes Petrucci at Hawthorne Ridge are also acknowledged for helping with subject recruitment and retainment. The authors would also like to acknowledge Andrew Bierman, Anna Murphy, Martin Overington, Brittany Wood, Robert Hamner, Roy Plummer, Sharon Lesage, Rebekah Mullaney, Dennis Guyon, and Sarah Hulse of the Lighting Research Center for their technical and editorial assistance. Mary S Rea, PhD, Jackie Petteys, and Kaitlyn Westfall of Russell Sage College are acknowledged for their assistance with data collection.

\section{Author contributions}

MGF conceived and designed the study, coordinated data collection, performed data and statistical analysis, and wrote the manuscript. BAP performed data collection and analyses, and participated in manuscript writing. AL participated in data collection and manuscript writing. GJ performed data analyses, assisted with manuscript writing, and reviewed the manuscript. MSR conceived and designed the study, and participated in manuscript writing. $\mathrm{PH}$ and $\mathrm{TH}$ were collaborators in the study, and participated in manuscript writing and review. All authors read and approved the final manuscript.

\section{Disclosure}

The authors report no conflicts of interest in this work.

\section{References}

1. nia.nih.gov [homepage on the Internet]. About Alzheimer's disease: Alzheimer's basics. National Institute on Aging; 2014 [updated May 22, 2014; cited May 22, 2014]. Available from: http://www.nia.nih. gov/alzheimers/topics/alzheimers-basics. Accessed July 20, 2014.

2. Mishima K, Hishikawa Y, Okawa M. Randomized, dim light controlled, crossover test of morning bright light therapy for rest-activity rhythm disorders in patients with vascular dementia and dementia of Alzheimer's type. Chronobiol Int. 1998;15(6):647-654.

3. Lyketsos CG, Lindell Veiel L, Baker A, Steele C. A randomized, controlled trial of bright light therapy for agitated behaviors in dementia patients residing in long-term care. Int J Geriatr Psychiatry. 1999; 14(7):520-525.

4. Yamadera H, Ito T, Suzuki H, Asayama K, Ito R, Endo S. Effects of bright light on cognitive and sleep-wake (circadian) rhythm disturbances in Alzheimer-type dementia. Psychiatry Clin Neurosci. 2000; 54(3):352-353.

5. Van Someren EJ, Kessler A, Mirmiran M, Swaab DF. Indirect bright light improves circadian rest-activity rhythm disturbances in demented patients. Biol Psychiatry. 1997;41(9):955-963.

6. Riemersma-van der Lek RF, Swaab DF, Twisk J, Hol EM, Hoogendijk WJ, Van Someren EJ. Effect of bright light and melatonin on cognitive and noncognitive function in elderly residents of group care facilities: a randomized controlled trial. JAMA. 2008;299(22):2642-2655.

7. Dowling GA, Hubbard EM, Mastick J, Luxenberg JS, Burr RL, Van Someren EJ. Effect of morning bright light treatment for restactivity disruption in institutionalized patients with severe Alzheimer's disease. Int Psychogeriatr. 2005;17(2):221-236.

8. Alessi CA, Martin JL, Webber AP, Cynthia KE, Harker JO, Josephson KR. Randomized, controlled trial of a nonpharmacological intervention to improve abnormal sleep/wake patterns in nursing home residents. $J$ Am Geriatr Soc. 2005;53(5):803-810.

9. Ancoli-Israel S, Martin J, Shochat T, Marler M. Morning light delays activity acrophase in demented elderly. Soc Light Treat Biol Rhythms. 2000;12:15.

10. Koyama E, Matsubara H, Nakano T. Bright light treatment for sleepwake disturbances in aged individuals with dementia. Psychiatry Clin Neurosci. 1999;53(2):227-229.

11. Lovell BB, Ancoli-Israel S, Gevirtz R. Effect of bright light treatment on agitated behavior in institutionalized elderly subjects. Psychiatry Res. 1995;57(1):7-12.

12. Satlin A, Volicer L, Ross V, Herz L, Campbell S. Bright light treatment of behavioral and sleep disturbances in patients with Alzheimer's disease. Am J Psychiatry. 1992;149(8):1028-1032.

13. Ancoli-Israel S, Martin JL, Gehrman P, et al. Effect of light on agitation in institutionalized patients with severe Alzheimer disease. Am J Geriatr Psychiatry. 2003;11(2):194-203.

14. Barrick AL, Sloane PD, Williams CS, et al. Impact of ambient bright light on agitation in dementia. Int J Geriatr Psychiatry. 2010; 25(10):1013-1021.

15. Colenda CC, Cohen W, McCall WV, Rosenquist PB. Phototherapy for patients with Alzheimer disease with disturbed sleep patterns: results of a community-based pilot study. Alzheimer Dis Assoc Disord. 1997; 11(3): 175-178.

16. Fontana Gasio P, Kräuchi K, Cajochen C, et al. Dawn-dusk simulation light therapy of disturbed circadian rest-activity cycles in demented elderly. Exp Gerontol. 2003;38(1-2):207-216. 
17. Sloane PD, Williams CS, Mitchell CM, et al. High-intensity environmental light in dementia: effect on sleep and activity. J Am Geriatr Soc. 2007;55(10):1524-1533.

18. Thapan K, Arendt J, Skene DJ. An action spectrum for melatonin suppression: evidence for a novel non-rod, non-cone photoreceptor system in humans. J Physiol. 2001;535(Pt 1):261-267.

19. Rea MS, Figueiro MG, Bullough JD, Bierman A. A model of phototransduction by the human circadian system. Brain Res Rev. 2005;50(2):213-228.

20. Brainard GC, Hanifin JP, Greeson JM, et al. Action spectrum for melatonin regulation in humans: evidence for a novel circadian photoreceptor. J Neurosci. 2001;21(16):6405-6412.

21. Figueiro MG, Rea MS. LEDs: Improving the sleep quality of older adults. Paper presented at the CIE Midterm Meeting and International Lighting Congress; May 18-21, 2005; Leon, Spain.

22. Figueiro MG, Eggleston G, Rea MS. Effects of Light Exposure on Behavior of Alzheimer's Patients - A Pilot Study: Proceedings from the 5th International Symposium on Light and Human Health, Orlando, FL, 3-5 November 2002. Palo Alto, CA: EPRI Lighting Research Office; 2002:151-156.

23. Figueiro MG. A proposed $24 \mathrm{~h}$ lighting scheme for older adults. Light Res Technol. 2008;40(2):153-160.

24. Meesters Y, Dekker V, Schlangen LJ, Bos EH, Ruiter MJ. Low-intensity blue-enriched white light (750 lux) and standard bright light (10,000 lux) are equally effective in treating SAD. A randomized controlled study. BMC Psychiatry. 2011;11:17.

25. Francis G, Bishop L, Luke C, Middleton B, Williams P, Arendt J. Sleep during the Antarctic winter: preliminary observations on changing the spectral composition of artificial light. J Sleep Res. 2008;17(3):354-360.

26. Smith MR, Eastman CI. Phase delaying the human circadian clock with blue-enriched polychromatic light. Chronobiol Int. 2009;26(4): 709-725.

27. Chodosh J, Edelen MO, Buchanan JL, et al. Nursing home assessment of cognitive impairment: development and testing of a brief instrument of mental status. J Am Geriatr Soc. 2008;56(11):2069-2075.

28. American Psychiatric Association. Diagnostic and Statistical Manual of Mental Disorders. 4th Ed. (DSM-IV). Arlington, VA: American Psychiatric Association; 2000

29. World Medical Association. World Medical Association Declaration of Helsinki. Ethical principles for medical research involving human subjects. JAMA. 2000;284(23):3043-3045.
30. Figueiro MG, Rea MS, Bullough JD. Does architectural lighting contribute to breast cancer? J Carcinog. 2006;5:20.

31. Zeitzer JM, Dijk DJ, Kronauer R, Brown E, Czeisler C. Sensitivity of the human circadian pacemaker to nocturnal light: melatonin phase resetting and suppression. J Physiol. 2000;526 Pt 3:695-702.

32. Figueiro MG, Hamner R, Bierman A, Rea MS. Comparisons of three practical field devices used to measure personal light exposures and activity levels. Light Res Technol. 2013;45(4):421-434.

33. Rea MS, Figueiro MG, Bierman A, Bullough JD. Circadian light. J Circadian Rhythms. 2010;8(1):2.

34. Rea MS, Bierman A, Figueiro MG, Bullough JD. A new approach to understanding the impact of circadian disruption on human health. J Circadian Rhythms. 2008;6:7.

35. Buysse DJ, Reynolds CF, Monk TH, Hoch CC, Yeager AL, Kupfer DJ. Quantification of subjective sleep quality in healthy elderly men and women using the Pittsburgh Sleep Quality Index (PSQI). Sleep. 1991; 14(4):331-338.

36. Morris JN, Fries BE, Morris SA. Scaling ADLs within the MDS. J Gerontol A Biol Sci Med Sci. 1999;54(11):M546-M553.

37. Alexopoulos GS, Abrams RC, Young RC, Shamoian CA. Cornell Scale for Depression in Dementia. Biol Psychiatry. 1988;23(3):271-284.

38. Cohen-Mansfield J, Marx MS, Rosenthal AS. A description of agitation in a nursing home. J Gerontol. 1989;44(3):M77-M84.

39. Figueiro MG, Hamner R, Higgins P, Hornick T, Rea MS. Field measurements of light exposures and circadian disruption in two populations of older adults. J Alzheimers Dis. 2012;31(4):711-715.

40. Hébert M, Martin SK, Lee C, Eastman CI. The effects of prior light history on the suppression of melatonin by light in humans. J Pineal Res. 2002;33(4):198-203.

41. Sloane PD, Figueiro MG, Garg S, et al. Effect of home-based light treatment on persons with dementia and their caregivers. Light Res Technol. Epub 2014 February 7.

42. Hickman SE, Barrick AL, Williams CS, et al. The effect of ambient bright light therapy on depressive symptoms in persons with dementia. $J$ Am Geriatr Soc. 2007;55(11):1817-1824.

43. Alzheimer's Association. 2012 Alzheimer's disease facts and figures. Alzheimers Dement. 2012;8(2):131-168.

44. McCurry SM, Pike KC, Vitiello MV, Logsdon RG, Larson EB, Teri L. Increasing walking and bright light exposure to improve sleep in community-dwelling persons with Alzheimer's disease: results of a randomized, controlled trial. J Am Geriatr Soc. 2011;59(8):1393-1402.
Clinical Interventions in Aging

\section{Publish your work in this journal}

Clinical Interventions in Aging is an international, peer-reviewed journal focusing on evidence-based reports on the value or lack thereof of treatments intended to prevent or delay the onset of maladaptive correlates of aging in human beings. This journal is indexed on PubMed Central, MedLine,

\section{Dovepress}

CAS, Scopus and the Elsevier Bibliographic databases. The manuscript management system is completely online and includes a very quick and fair peer-review system, which is all easy to use. Visit http://www.dovepress. com/testimonials.php to read real quotes from published authors. 